\title{
Lower fat and better quality diet therapy for children with pharmacoresistant epilepsy
}

\author{
Jung-Rim Yoon, MD, Heung Dong Kim, MD, PhD, Hoon-Chul Kang, MD, PhD \\ Division of Pediatric Neurology, Department of Pediatrics, Pediatric Epilepsy Clinic, Epilepsy Research Institute, Severance Children's Hospital, Yonsei University \\ College of Medicine, Seoul, Korea
}

The ketogenic diet (KD) is an established, effective, nonpharmacologic treatment for children with pharmacoresistant epilepsy. Although the KD is the most well-established dietary therapy for epilepsy, it is too restrictive and is associated with serious complications; therefore, alternative lower-fat diets, including a modified Atkins diet and low-glycemic index diet, have been developed. Recent ongoing clinical evidence suggests that other dietary therapies have an efficacy almost comparable to that of the KD. In addition, a diet rich in polyunsaturated fatty acids appears to increase the efficacy of diet therapy and reduce the complications of a high-fat diet. Here, we review the systematic information about lower-fat diets and better-quality dietary therapies and the current clinical status of each of these dietary approaches.

Key words: Ketogenic diet, Atkins diet, Low-glycemic index diet, Polyunsaturated fatty acid, Epilepsy
Corresponding author: Hoon-Chul Kang, MD, PhD Department of Pediatrics, Severance Children's Hospital, Yonsei University College of Medicine, 50 Yonsei-ro, Seodaemun-gu, Seoul 120-752, Korea Tel: +82-2-2228-2075

Fax: $+82-2-393-9118$

E-mail: hip00207@yuhs.ac

Received: 18 February, 2013

Accepted: 4 June, 2013

\section{Introduction}

Fasting as a treatment for epilepsy was described in the New Testament of the Bible, and the earliest scientific approach was introduced by Geyelin in the $1920 \mathrm{~s}^{11}$. Since its resurgence in the mid-1990s, the ketogenic diet (KD) has been used worldwide, including Korea, and is recognized as a valuable adjunct therapeutic modality ${ }^{2)}$. The classic KD, with a nonfat-to-fat ratio of 1:4, necessitates a regimen that is high in fat, adequate in protein $(1 \mathrm{~g} / \mathrm{kg} /$ day), and low in carbohydrates. Since $1 \mathrm{~g}$ of fat produces $9 \mathrm{cal}$ and $1 \mathrm{~g}$ of protein or carbohydrate produces $4 \mathrm{cal}$, when the ratio of constituents are calculated on a calorie scale, the ratio appears more exaggerated ${ }^{3)}$ (Fig. 1). The KD is difficult to maintain due to its restrictive nature, and it has potentially serious complications. We require alternative diet therapies, including a modified Atkins diet (MAD), low-glycemic index diet (LGID), and a diet rich in polyunsaturated fatty acids (PUFAs), that are safer and more convenient to use and can improve the outcome.

Here, we discuss the systematic information and current clinical status of each of these dietary approaches.

\section{What is the KD?}

Copyright (C) 2013 by The Korean Pediatric Society

This is an open-access article distributed under the terms of the Creative Commons Attribution NonCommercial License (http://creativecommons.org/ licenses/by-nc/3.0/) which permits unrestricted noncommercial use, distribution, and reproduction in any medium, provided the original work is properly cited. 

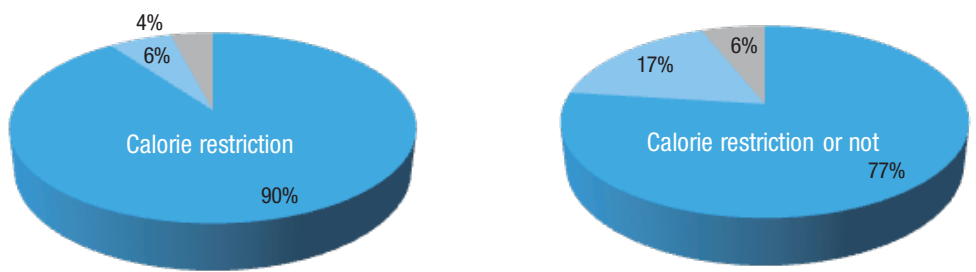

A

Ketogenic diet (\% cal) - Fat $\square$ Protein $\square$ Carbohydrate

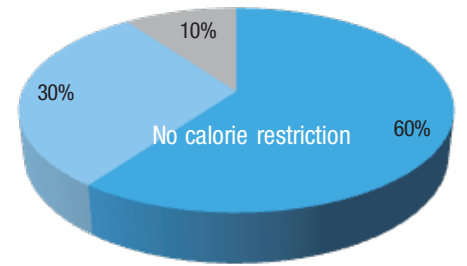

Low-glycemic index diet (\% cal)

C Fat $\square$ Protein $\square$ Carbohydrate (low glycemic index)
Modified Atkins diet (\% cal)

Fat $\square$ Protein $\square$ Carbohydrate

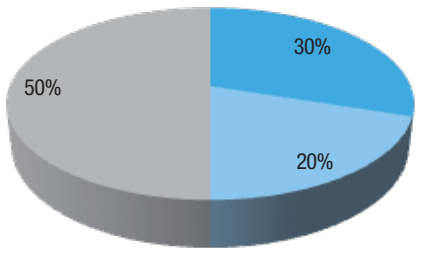

Regular diet (\% cal)

D Fat Protein Carbohydrate

Fig. 1. The ratio of diet composition of the classic ketogenic diet (A0, a modified Atkins diet (B), and lowglycemic index diet (C), compared to that of a regular diet (D) on a calorie scale.

gastrointestinal (GI) problems ${ }^{5,6)}$. However, the efficacy of seizure control in these diets is similar ${ }^{6}$. There is a large clinical database of evidence on the efficacy of the $\mathrm{KD}$, and most studies show similar outcomes ${ }^{7-10)}$. Our experience using the classic KD for 199 children with pharmacoresistant epilepsy suggests that after 12 months on the KD, approximately $41 \%$ of the children had at least a $50 \%$ reduction in the number of seizures and 25\% of children were seizure-free ${ }^{8)}$. Although the efficacy of the $\mathrm{KD}$ is not maintained in all patients after they discontinue the diet, the effects of the KD persist in most patients as seen by an alleviation of symptoms that appeared in the pre-KD administration period ${ }^{11)}$. The widespread acceptance of the KD has ended the debate about its efficacy. However, despite this, many families and physicians are reluctant to use the $\mathrm{KD}$ because of its inherent difficulties as well as the various complications that can stem from the nutritionally unbalanced composition of the KD.

As a safer and more comfortable diet therapy, MAD or LGID could be considered. Fat is ketogenic and carbohydrate is antiketogenic. Therefore, these diets induce a state of ketosis by providing high fat and low carbohydrate, although they involve intake of large amounts of protein, which is restricted in the conventional $\mathrm{KD}^{8-10)}$. Therefore, a more tolerable diet with a reduced risk of the complications is desired.

\section{Complications of KD}

Poor tolerability and the potentially serious complications of an unbalanced diet should be considered. Some adverse events occur within a few days or a month of diet initiation; other events may occur after several months. According to our experience using $\mathrm{KD}$ therapy at Severance Hospital ${ }^{12}$, the most common early onset complication is GI disturbances such as nausea/vomiting, diarrhea, and constipation. These complications are sometimes associated with gastritis and fat intolerance. Other early onset complications, in order of frequency, are hypertriglyceridemia; transient hyperuricemia, hypercholesterolemia, various infectious diseases, symptomatic hypoglycemia, hypoproteinemia, hypomagnesemia, repetitive hyponatremia, low concentrations of high-density lipoprotein, lipoid pneumonia due to aspiration, hepatitis, acute pancreatitis, and persistent metabolic acidosis. Late-onset complications also include osteopenia, renal stones, cardiomyopathy, secondary hypocarnitinemia, and iron-deficiency anemia. Most early and late-onset complications are transient and can be successfully managed by careful follow-up and conservative strategies. However, 22 of 129 patients (17.1\%) stopped the $\mathrm{KD}$ due to various kinds of serious complications and 4 of 129 patients (3.1\%) died during the KD: 2 from sepsis, 1 from cardiomyopathy, and 1 from lipoid pneumonia ${ }^{12)}$. The $\mathrm{KD}$ is restrictive and has various complications; therefore, the use of other possible alternative lower-fat and better-quality diets, including the MAD, LGID, and a PUFA-rich diet, is encouraged.

\section{MAD}

The Atkins diet was created in the 1970s by the late Dr. Robert C. Atkins, as a means to combat obesity. It is nearly a balanced 
diet (60\% fat, 30\% protein, and 10\% carbohydrate by weight, or 77\% fat, 17\% protein, and 6\% carbohydrate by calorie) compared with the KD (80\% fat, 15\% protein, and 5\% carbohydrate by weight, or 90\% fat, 6\% protein, 4\% carbohydrate by calorie) (Fig. 1). The protocol of an MAD suggests that carbohydrates should be restricted to $10 \mathrm{~g}$ per day in the first month ${ }^{13)}$. If a child has difficulty with this initial restricted amount, then an increase of 5 $\mathrm{g}$ per day is allowed for at least 1 month, to a maximum of $10 \%$ carbohydrates per day (by weight). Multivitamins, calcium, and vitamin $\mathrm{D}_{2}$ were administered as supplements. We recommended that patients consume $75 \%$ of their recommended daily calorie intake, which is different from the Hopkins protocol and similar to the conventional $\mathrm{KD}^{14)}$. This recommendation was based on our previous experience, albeit anecdotal, of more favorable outcomes using the MAD with restricted calories ${ }^{14)}$. In addition, we recommended that patients frequently measure urine ketone levels at home, especially when seizures occur or the frequency of the seizures increases. The patients' tolerability of the diet and complications were assessed using various methods, including laboratory evaluations, on a regular basis (Table 1).

The most previous series showed striking similarities to large prospective and retrospective studies of the classic $\mathrm{KD}$; however, we found a trend for a higher incidence of responders (>50\% seizure reduction) in the $\mathrm{KD}$ group ${ }^{15)}$, and the $\mathrm{KD}$ and MAD need to be compared in a well-controlled, randomized study in order to ensure a good evidence base for decisions in the usual situations. In contrast with the efficacy, there was no doubt about the tolerability of the MAD ${ }^{15-19)}$. Therefore, a MAD diet is strongly recommended for patients who experience problems with the conventional $\mathrm{KD}$, which was shown to be effective but too restrictive, as well as for patients who require a long-term diet therapy trial due to the presence of glucose transporter type 1deficiency syndrome or selected mitochondrial diseases.

\section{LGID}

The glycemic index provides a measure of how quickly levels of glucose in the blood increase after consuming a particular type of food, relative to the consumption of pure glucose. Glycemic indices of various food are presented in Table 2. The LGID would reduce blood glucose by altering the quality and types of carbohydrates consumed. The LGID for refractory epilepsy was first reported by Pfeifer and Thiele ${ }^{20)}$ in 2005. Glucose has a glycemic index of 100. In the LGID, foods are limited to those with a glycemic index of less than 50 . Total carbohydrates are gradually decreased to 40-60 gm/day ( 10\% of daily calories). Approximately $20-30 \%$ of calories are from protein and 60-70\% are from fat (Fig. 1). Muzykewicz et al. ${ }^{21)}$ reported that a $>50 \%$ reduction in seizure frequency in 76 patients was observed in $42 \%, 50 \%, 54 \%$, and $66 \%$ of patients with follow-up available at $1,3,6,9$, and 12 months. The LGID seems to have efficacy similar to that seen in the KD observational studies, but there are no randomized controlled trials studies comparing the $\mathrm{KD}$ with the LGID. Thus, further studies are needed to evaluate and compare the efficacies of these diets.

Table 1. The suggested protocol of a modified Atkins diet at Severance Hospital

Carbohydrates were restricted to $10 \mathrm{~g}$ per day for the first month.

Permitted to increase by only $5 \mathrm{~g}$ per day up to $10 \%$ carbohydrate by weight with an interval of at least one month.

$\mathrm{L}$-carnitine, multivitamins, and calcium, and vitamin $\mathrm{D}_{2}$ were given as supplements.

Calories are recommended to be restricted into $75 \%$ of recommended daily intake.

Seizure diary, blood or urine ketones semiweekly, and weight mont hly, height every 6 months

Medication changed to tablet or sprinkle (non liquid) preparations

All the patients had been advised to remain at the hospital for 3 or 4 days to ensure adequate diet adaptation to have their blood ketosis carefully monitored.

Low-carbohydrate, store-bought products (e.g., shakes, candy bars, and baking mixes) discouraged.

We recommended a trial of the diet for at least 2 months, even in the face of unfavorable results.

Table 2. Glycemic indices of various foods

\begin{tabular}{lll}
\hline Classification & \multicolumn{1}{c}{ Gl range } & \multicolumn{1}{c}{ Example } \\
\hline Low Gl & 55 or less & $\begin{array}{c}\text { Most fruits and vegetables (except potatoes and watermelon), grainy breads, pasta, legumes/pulses, milk, yoghurt, products extremely } \\
\text { low in carbohydrates (some cheeses, nuts), fructose } \\
\text { Medium Gl }\end{array}$ \\
$\begin{array}{l}\text { Whole wheat products, basmati rice, sweet potato, table sugar } \\
\text { High Gl }\end{array} 70$ and above & $\begin{array}{l}\text { Corn flakes, rice krispies, baked potatoes, watermelon, croissants, white bread, extruded breakfast cereals, most white rices, straight } \\
\text { glucose }\end{array}$ \\
\hline
\end{tabular}

Gl, glycemic index. 


\section{PUFA-enriched diet}

Fatty acids are the most important contributor to calories in the KD and are important for all aspects of efficacy and complications of the $\mathrm{KD}^{12)}$. Unfortunately, however, most fatty acids in the conventional KD are saturated or monounsaturated because the diet is based primarily on butter, cream, and olive $\mathrm{oil}^{22)}$. PUFAs are believed to favorably affect neuronal and cardiovascular function ${ }^{23)}$.

There are 2 groups of PUFAs: n-3 and n-6 PUFAs. Among dietary n-3 PUFAs, alpha-linoleic acid (ALA) is found in flax seeds, walnuts, and almonds, and eicosapentaenoic acid and docosahexanoic acid are found in marine fish and seals. The primary n-3 PUFA in the brain is DHA, and it can be obtained directly from the diet or can be synthesized from $\mathrm{ALA}^{23)}$. In addition, the conversion of ALA to DHA can be optimized when ALA is coadministered with the n- 6 fatty acid LA at a 1:4 ratio, as observed in rat brains ${ }^{24)}$. The $n-6$ PUFAs are found in vegetable oils such as canola and corn oil. Canola oil also has large amounts of n-3 and a 1:4 ratio of n-3 to n-6.

Recent evidence suggests that the KD may be useful in the treatment of epilepsy owing to the presence of PUFAs ${ }^{25}$. PUFAs stimulate mitochondrial biogenesis and enhance energy reserves, leading to stabilized synaptic function and improved seizure control. Clinically, Fraser et al. ${ }^{26)}$ described 9 children on the KD whose elevated blood PUFAs were correlated with improved seizure control. Recently, we reported the case of a 7-year-old boy with Lennox-Gastaut syndrome combined with mitochondrial respiratory chain complex I deficiency, whose medically intractable seizures were successfully controlled with a PUFA-enriched $\mathrm{MAD}$ without any significant adverse events. The diet consists of canola oil and diverse menu items such as fish and nuts (instead of olive oil) and has an ideal 1:2.8 ratio of n-3 to n-6 (Table 3). In addition, some studies suggest that $n-3$ and n-6 PUFAs together may be clinically important for preventing cardiovascular disorders ${ }^{27}$. The ratio of n-3 to n-6 PUFAs is known to be important for maintaining cardiovascular health ${ }^{27)}$. In our patient, a modified diet therapy with canola oil and a diverse menu resulted in an ideal ratio of n-3 to n-6 PUFAs of 1:2.8, and the diet is expected to decrease the risk for cardiovascular complications, even with long-term maintenance of the high-fat diet.

Table 3. Constituents of fatty acids in a modified Atkins diet of $1,200 \mathrm{kcal}$ and ratio of fat to nonfat of 1.7:1 on pre- and post-PUFA-enriched diet Pre-PUFA-enriched diet Post-PUFA-enriched diet Saturated fatty acids $\quad 17.9 \mathrm{~g}$ (18.4\% weight) $14.4 \mathrm{~g}$ (14.5\% weight) Monounsaturated fatty acids $61.7 \mathrm{~g}$ (63.5\% weight) $54.7 \mathrm{~g}$ (54.9\% weight) Polyunsaturated fatty acids $17.6 \mathrm{~g}$ (18.1\% weight) $30.5 \mathrm{~g}$ (30.6\% weight) Ratio of n-3:n-6 PUFAs $1: 18.6$ $1: 2.8$

PUFAs, polyunsaturated fatty acids.

\section{Conclusions}

The ability of children with pharmacoresistant epilepsy to tolerate the MAD or LGID is encouraging, suggesting that this diet can replace the conventional $\mathrm{KD}$, especially in patients who require long-term diet therapy trial due to the presence of glucose transporter type 1-deficiency syndrome or selected mitochondrial diseases, as well as in patients who experience problems with the conventional $\mathrm{KD}$, which is effective but too restrictive. PUFAenriched diet therapy is also expected to increase the efficacy of diet therapy and reduce complications of a high-fat diet in children with refractory epilepsy. However, there are few evidence-based studies that compare the conventional KD with the MAD, LGID, or PUFA-enriched diet, and such further studies are needed.

\section{Conflict of interest}

No potential conflict of interest relevant to this article was reported.

\section{Acknowledgments}

This work was supported by National Research Foundation grant funded by the Korea government (MEST, 2010-0020353).

\section{References}

1. Geyelin HR. Fasting as a method for treating epilepsy. Med Rec 1921;99:1037-9.

2. Kossoff EH, McGrogan JR. Worldwide use of the ketogenic diet. Epilepsia 2005;46:280-9.

3. Kossoff EH. More fat and fewer seizures: dietary therapies for epilepsy. Lancet Neurol 2004;3:415-20.

4. Swink TD, Vining EP, Freeman JM. The ketogenic diet: 1997. Adv Pediatr 1997;44:297-329.

5. Huttenlocher PR, Wilbourn AJ, Signore JM. Medium-chain triglycerides as a therapy for intractable childhood epilepsy. Neurology 1971;21:1097-103.

6. Schwartz RH, Eaton J, Bower BD, Aynsley-Green A. Ketogenic diets in the treatment of epilepsy: short-term clinical effects. Dev Med Child Neurol 1989;31:145-51.

7. Thiele EA. Assessing the efficacy of antiepileptic treatments: the ketogenic diet. Epilepsia 2003;44 Suppl 7:26-9.

8. Kang HC, Kim YJ, Kim DW, Kim HD. Efficacy and safety of the ketogenic diet for intractable childhood epilepsy: Korean multicentric experience. Epilepsia 2005;46:272-9.

9. Miranda MJ, Turner Z, Magrath G. Alternative diets to the classical ketogenic diet: can we be more liberal? Epilepsy Res 2012;100:27885.

10. Cross JH, Neal EG. The ketogenic diet: update on recent clinical trials. Epilepsia 2008;49 Suppl 8:6-10.

11. Hemingway C, Freeman JM, Pillas DJ, Pyzik PL. The ketogenic diet: 
a 3- to 6-year follow-up of 150 children enrolled prospectively. Pediatrics 2001;108:898-905.

12. Kang HC, Chung DE, Kim DW, Kim HD. Early- and late-onset complications of the ketogenic diet for intractable epilepsy. Epilepsia 2004;45:1116-23.

13. Kossoff EH, McGrogan JR, Bluml RM, Pillas DJ, Rubenstein JE, Vining EP. A modified Atkins diet is effective for the treatment of intractable pediatric epilepsy. Epilepsia 2006;47:421-4.

14. Kim YM, Vaidya VV, Khusainov T, Kim HD, Kim SH, Lee EJ, et al. Various indications for a modified Atkins diet in intractable childhood epilepsy. Brain Dev 2012;34:570-5.

15. Porta N, Vallee L, Boutry E, Fontaine M, Dessein AF, Joriot S, et al. Comparison of seizure reduction and serum fatty acid levels after receiving the ketogenic and modified Atkins diet. Seizure 2009;18: 359-64.

16. Miranda MJ, Mortensen M, Povlsen JH, Nielsen H, Beniczky S. Danish study of a modified Atkins diet for medically intractable epilepsy in children: can we achieve the same results as with the classical ketogenic diet? Seizure 2011;20:151-5.

17. Tonekaboni SH, Mostaghimi P, Mirmiran P, Abbaskhanian A, Abdollah Gorji F, Ghofrani M, et al. Efficacy of the Atkins diet as therapy for intractable epilepsy in children. Arch Iran Med 2010;13: 492-7.

18. Weber S, Molgaard C, Karentaudorf, Uldall P. Modified Atkins diet to children and adolescents with medical intractable epilepsy. Seizure 2009;18:237-40.
19. Kang HC, Lee HS, You SJ, Kang du C, Ko TS, Kim HD. Use of a modified Atkins diet in intractable childhood epilepsy. Epilepsia 2007;48:182-6.

20. Pfeifer HH, Thiele EA. Low-glycemic-index treatment: a liberalized ketogenic diet for treatment of intractable epilepsy. Neurology 2005;65:1810-2.

21. Muzykewicz DA, Lyczkowski DA, Memon N, Conant KD, Pfeifer $\mathrm{HH}$, Thiele EA. Efficacy, safety, and tolerability of the low glycemic index treatment in pediatric epilepsy. Epilepsia 2009;50:1118-26.

22. Dahlin M, Hjelte L, Nilsson S, Amark P. Plasma phospholipid fatty acids are influenced by a ketogenic diet enriched with $n-3$ fatty acids in children with epilepsy. Epilepsy Res 2007;73:199-207.

23. Taha AY, Burnham WM, Auvin S. Polyunsaturated fatty acids and epilepsy. Epilepsia 2010;51:1348-58.

24. Yehuda S, Carasso RL, Mostofsky DI. Essential fatty acid preparation (SR-3) raises the seizure threshold in rats. Eur J Pharmacol 1994; 254:193-8.

25. Bough KJ, Rho JM. Anticonvulsant mechanisms of the ketogenic diet. Epilepsia 2007;48:43-58.

26. Fraser DD, Whiting S, Andrew RD, Macdonald EA, Musa-Veloso K, Cunnane SC. Elevated polyunsaturated fatty acids in blood serum obtained from children on the ketogenic diet. Neurology 2003;60: 1026-9.

27. Lavie CJ, Milani RV, Mehra MR, Ventura HO. Omega-3 polyunsaturated fatty acids and cardiovascular diseases. J Am Coll Cardiol 2009;54:585-94. 